\title{
Thyroid Hemiagenesis Presenting As Hypothyroidism - A Rare Case Report
}

\author{
Vishwanath TT, Singh S, Shetty SMCC, Apoorva C
}

Department of Radiodiagnosis, JSS Medical College, Mysore, Karnataka, India

\begin{abstract}
Thyroid hemiagenesis is a very rare abnormality, in which one of the thyroid lobes fails to develop embryologically. Most of the patients are detected incidentally and have an associated thyroid disease. The true prevalence of the thyroid hemiagenesis is still not known but literature reports incidence in $0.02 \%$ of population. We report a rare case of hemiagenesis of left lobe of thyroid and isthmus with associated hypothyroidism in a 28 years old female with review of literature.
\end{abstract}

Keywords: Hypothyroidism, Thyroid hemiagenesis, Ultrasound

\section{Introduction}

Thyroid hemiagenesis is a rare congenital developmental abnormality in one lobe of thyroid due to failure in the normal development. ${ }^{1}$ First described by Handsfield John in the year 1866. The thyroid gland begins to develop as a median thickening of endoderm on the floor of pharynx between first and second pharyngeal pouches. This area later invaginates to form the median diverticulum which appears later. The diverticulum later grows caudally as a bifurcating tubular duct from which the lateral lobes and isthmus develop. ${ }^{2}$ The cause of unilateral agenesis is not known, but

Correspondence to: Dr. Simran Singh MBBS, Resident, Department of Radiodiagnosis, JSS Medical College, Mysore, Karnataka, India-570004

Email:- dr.simrans@gmail.com it is believed to be arising from the failure of the original anlage to become bilobed and spread out laterally in both halves. ${ }^{1}$

\section{Case Report}

A 28 year old female patient presented with a history of hypothyroidism and on thyroxin $50 \mathrm{mcg}$ tablets from past 10 years for regular thyroid scan to rule out any focal lesions. No previous histories of any surgery, family history of any thyroid disorder were given.

Ultrasound revealed complete absence of left lobe of thyroid and partial left sided absence of isthmus (Fig. 1) while the right lobe of thyroid was normal and measured $4.1 \times 3.5 \mathrm{x}$ $3.6 \mathrm{cms}$ (volume $\sim 25 \mathrm{ml}$ ) with normal echogenicity (Fig. 2). No focal nodules, cystic changes or calcifications seen within the right lobe of thyroid. 


\section{Discussion}

The congenital absence of one lobe of thyroid is rare condition of an unknown etiology. It has been postulated that it occurs due to result of failure of thyroid analage to become bilobed followed by unilateral migration. ${ }^{811}$ Most of the cases which are diagnosed showed female predominance with a ratio of 3:1 and also predominance of left lobe is far more than the right lobe with a ratio of $4: 1{ }^{10}$ The same is seen in our case where there is complete absence of left lobe along with the isthmus.

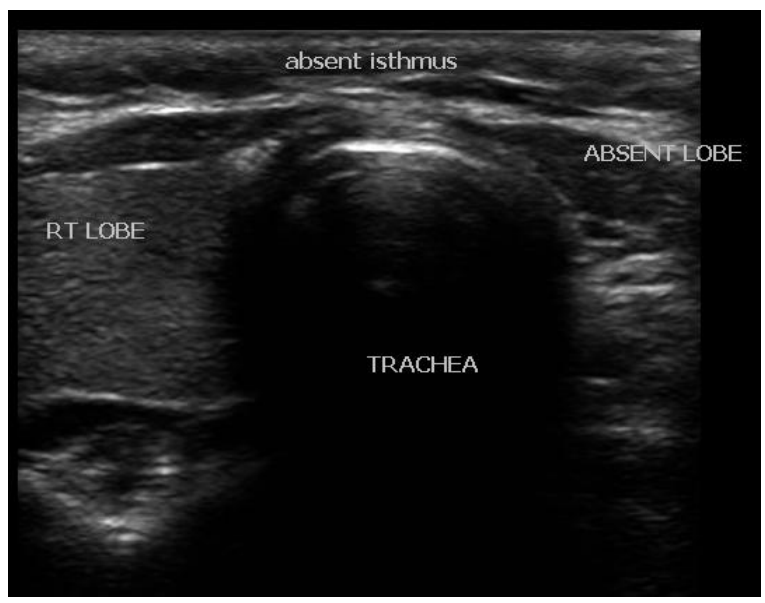

Fig. 1: Grey scale image showing complete absence of left lobe of thyroid and partial agenesis of isthmus.

The actual incidence of the hemiagenesis is unknown as most patients worldwide are admitted with a suspicion of other thyroid abnormalities like benign adenoma, multinodular goiter, hyper and hypothyroidism (as in our case) and hemiagenesis is seen as an incidental finding. $10-13$

Thyroid agenesis should be suspected in all the cases where there is no palpable thyroid tissue felt on one or either side of the neck. Radiological investigations hold the key role in diagnosing this condition. A definitive diagnosis is made by ultrasound and CT, ultrasound examination can easily establish the diagnosis in suspected cases. ${ }^{5}$ Ultrasound examination is far cheaper, easy to perform and above all there are no radiation hazards. Thyroid scintigraphy may also be helpful in differentiating hemiagenesis from a suppressed lobe. It will show total absence of uptake in congenital hemiagenesis, but the activity will reappear in suppressed lobe. ${ }^{3}$

The lobe of the thyroid which is functioning can be site of pathological changes similarly to a normally developed gland and it may present with spectrum of diseases which include, follicular adenoma, colloid goiter, multinodular goiter, thyroiditis, primary hyperthyroidism and hypothyroidism. ${ }^{4-6}$ Unilateral absence of superior and inferior thyroid artery, right sided aortic arch is other associated anomalies with hemiagenesis of the thyroid. ${ }^{7}$

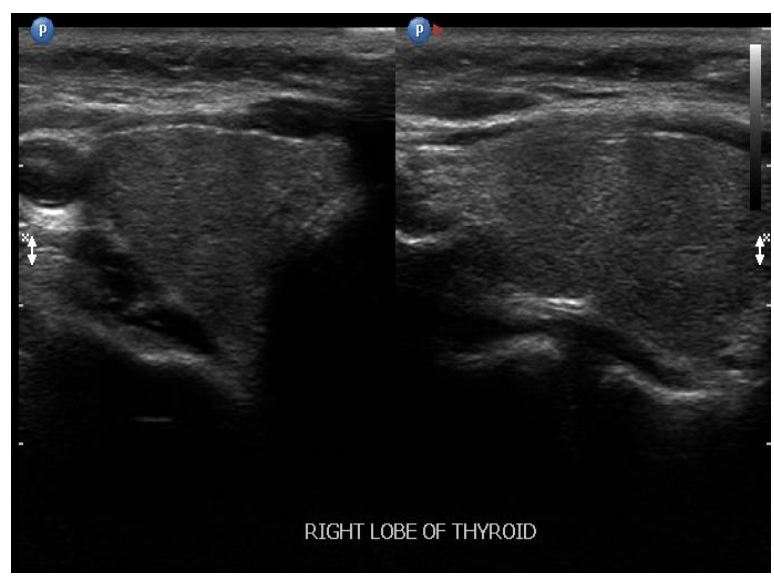

Fig. 2: Grey scale ultrasound image showing the normal right lobe of thyroid.

Although our patient had hypothyroidism, the most common disease associated with the remaining thyroid is hyperthyroidism. Hypothyroidism remains an unusual complication of the thyroid hemiagenesis with an uncertain etiology. ${ }^{10,14,15}$ Thyroid hormone deficiency is mainly due to 
peripheral and central nervous system dysfunctions that makes it even more important to diagnose hypothyroidism. ${ }^{12,16}$

We conclude that thyroid hemiagenesis and diffuse or focal thyroid disease in the existing thyroid lobe can be demonstrated by ultrasound well and stands as investigation of choice, further associated anomalies are delineated by CT and scintigraphy helps for differentiation between hemiagenesis and suppressed thyroid lobe.

\section{References}

1. Greening WP, Sarker SK, Osborne MP. Hemiagenesis of thyroid gland.Br J Surg $1980 ; 67: 446-8$

2. Warwick R, editor. Williams PL. Gray's Anatomy, 36 the edition. Edinburgh: Churchill Livingstone, 1980; 198.

3. Hamburger JI, Hamburger SW. Thyroidal hemiagenesis. Arch Surg 1970; 100:319-20.

4. Melnick JC, Stemkowski PE. Thyroid hemiagenesis (hockey stick sign): a review of the world literature and report of four cases. J Clin Endocrinol 1981; 52:247-51.

5. McHenry CR, Walfish PG, Rosen IB, Lawrence AM, Paloyan E. Congenital thyroid hemiagenesis. Am Surg1995; 61:634-9.

6. Burman KD, Adler RA, Wartofsky L. Hemiagenesis of thyroid gland. Am J Med 1975; 58:143-5.

7. Duh Q, Ciulla T, Clark OH. Primary parathyroid hyperplasia associated with thyroid hemiagenesis and the agenesis of the isthmus. Surgery 1994; 115:257-63.

8. McHenry CR, Walfish PG, Rosen IB, et al. Congenital thyroid hemiagenesis. Am Surg 1995; 61: 634-638.

9. Burman KD, Adler RA, Wartofsky L. Hemiagenesis of the thyroid gland. Am J Med 1975; 58: 143-146.

10. Shaha AR, Gujarati R. Thyroid hemiagenesis. J Surg Oncol 1997; 65: 137-140.

11. Greening WP, Sarker SK, Osborne MP. Hemiagenesis of the thyroid gland. $\mathrm{Br} \mathbf{J}$ Surg ; 67: 446-448.

12. YÝldÝz M, Bozkurt MK. Thyroid hemiagenesis. J Adolescent Health 2003; 33: 291-292.

13. Karabay N, Comlekci A, Canda MS, et al. Thyroid hemiagenesis with multinodular goiter: A case report and review of the literature. Endocrine Journal 2003; 50: 409-413.

14. Konno N, Kanaya A. Thyroid hemiagenesis associated with the right aortic arch. J Endocrinol Invest 1988; 11 : 685-687.

15. Melnick JC, Stemkowski PE. Thyroid hemiagenesis (hockey stick sign): A review of the world literature and a report of four cases. J Clin Endocrinol Metab 1981; 52: 247-251.

16. Ozata Mzkardeß A, GŸndoÛan MA. Effects of acute hypothyroidism on brainstem auditory evoked potentials (BAEPs). Tr J of Medical Sciences 1998; 28: 133-137. 